\title{
Analysis of means-end chain data in marketing research
}

Received: (in revised form): 25th September, 2006

\section{Eugene Kaciak}

$\mathrm{PhD}$, is Associate Professor of Management at Brock University in St Catharines, Ontario, Canada. He earned both his MSc in Economics (1973) in the field of Management Science and his PhD in the field of Econometrics (1977) at Warsaw School of Economics (Poland). He taught for more than 30 years at various business schools in Poland (Warsaw School of Economics), Algeria (University of Algiers) and Canada (University of Alberta, Laurentian University and Brock University). He published articles in a number of scientific journals, including Journal of Marketing Research, Journal of International Consumer Marketing and Revue Internationale PME.

\section{Carman W. Cullen}

$\mathrm{PhD}$, Associate Professor of Marketing, is a three-time winner of the Faculty of Excellence award in the Faculty of Business at Brock University. He has an extensive background in consumer research and retailing, both as practitioner and as a consultant. He has offered numerous seminars and conducted research throughout North America and Europe with a most recent focus on wine marketing.

\begin{abstract}
Means-End Chain Theory has been developed in order to understand how consumers link attributes $(A)$ of products with particular consequences $(C)$, and how these consequences satisfy their personal values $(V)$. The associations in the mind of the consumer between $A$ 's, $C$ 's and $V$ 's are labelled means-end chains (MEC). These chains are often seen as a representation of the basic drive that motivates consumer behaviour. Numerous studies have shown that techniques using MEC are suitable for a wide range of marketing applications. But there is no agreement among researchers as to the way MEC observations should be analysed. In this paper, we review methods of analysis of such observations and suggest the most appropriate procedures. Data from a study of smokers' perceptions of cigarettes in a European city are used for illustration purposes.

Journal of Targeting, Measurement and Analysis for Marketing (2006) 15, 12-20. doi:10.1057/palgrave.jt.5750028
\end{abstract}

\section{INTRODUCTION}

Means-End Chain Theory ${ }^{1-6}$ has been developed in order to understand how consumers link attributes $(A)$ of products with particular consequences $(C)$, and how these consequences satisfy their personal values $(V)$. The associations in the mind of the consumer between $A$ 's, $C$ 's and $V$ s are labelled means-end chains (MEC). These chains are often seen as a representation of the basic drive that motivates consumer behaviour. A comprehensive history of the MEC approach is presented by Olson and Reynolds. ${ }^{7}$

Correspondence: Eugene Kaciak, Faculty of Business, Brock University, St Catherines, Ontario L2S 3A1 Canada.

Tel: + 19056885550 ext 3902;

Fax: + 19056889779 ;

Email: ekaciak@brocku.ca
Numerous studies have shown that techniques using MEC are suitable for a wide range of marketing applications. But there is no agreement among researchers as to the way MEC observations should be analysed. In this paper, we will review methods of analysis of such observations and suggest the most appropriate procedures.

\section{COLLECTING MEC DATA}

Let us assume that content analysis of $n$ questionnaires has generated $a_{i}$ attributes $A_{i}, c_{j}$ consequences $C_{j}$ and $v_{k}$ values $V_{k}$, where $a_{i}+c_{j}+v_{k}=q$. Let $p$ be a number of $A-C-V$ connections (called ladders) provided by $n$ respondents, and let us assume, for the sake of 
simplicity, that each of these ladders has three levels (the procedures that follow are easily adaptable to other numbers of levels as well).

\section{Ladder matrix}

Let $L=\left[l_{r s}\right]$ be a $p \times q$ matrix with $l_{r s}$ defined as follows: $l_{r s}=1$, if the sth category was present in the $r$ th ladder among all the ladders that have been elicited from the $n$ respondents, otherwise $l_{r s}=0(r=1,2, \ldots, p ; s=1,2, \ldots, q)$.

\section{Summary implication matrix (SIM)}

The individual ladders in $L$ can be aggregated across all the respondents and presented in the form of a summary implication matrix or SIM. ${ }^{8}$ The elements of a SIM represent the number of times each category (the attribute, consequence or value) leads (directly or indirectly) to another category. The following procedure may be used to convert matrix $L$ into a SIM:

Step 1: Partition matrix $L$ into three submatrices, $A, C$ and $V$, whose columns correspond to the attribute, consequence and value categories, respectively. Matrix $A$ is a $p \times a_{i}$ matrix, $C$ is a $p$ $\times c_{j}$ matrix and $V$ is a $p \times v_{k}$ matrix. Thus, $L=[A$ $C V$. A typical row of $L$ will contain all 0 's except three 1's. A respondent may contribute more than one row to $L$.

Step 2: Calculate $L^{\prime} L$, which is a (symmetric) summary implication matrix or SIM of size $q=a_{i}+c_{j}+v_{k}$, and can be represented as follows:

$$
S I M=L^{\prime} L=\begin{array}{lll}
A^{\prime} A & A^{\prime} C & A^{\prime} V \\
C^{\prime} A & C^{\prime} C & C^{\prime} V \\
V^{\prime} A & V^{\prime} C & V^{\prime} V
\end{array}
$$

The elements of an $a_{i} \times c_{j}$ matrix $A^{\prime} C$ represent the number of direct connections in the ladders between each pair of attribute and benefit categories. The elements of a $c_{j} \times v_{k}$ matrix $C^{\prime} V$ describe the number of direct connections between each pair of consequence and value categories. The elements of an $a_{i} \times v_{k}$ matrix $A^{\prime} V$ correspond to the number of indirect connections between each pair of attribute and value categories. The matrices $A^{\prime} A, C^{\prime} C$ and $V^{\prime} V$ show on their main diagonals the number of times their corresponding categories are present in the $p$ ladders.
The matrices $A^{\prime} C, A^{\prime} V$ and $C^{\prime} V$ may also form an asymmetric summary implication matrix or SIM $M_{\text {asymm }}$, if arranged as follows:

$$
S I M_{\text {asymm }}=\begin{array}{ll}
A^{\prime} C & A^{\prime} V \\
0 & C^{\prime} V
\end{array}
$$

where 0 is a $c_{j} \times c_{j}$ matrix containing all zeros. The $S I M_{\text {asymm }}$ is a $\left(a_{i}+c_{j}\right) \times\left(c_{j}+v_{k}\right)$ matrix whose size is smaller than that of the (symmetric) SIM and, thus, it is a preferred choice for data presentation.

\section{Illustration}

We illustrate all procedures with data from a study of smokers' perceptions of cigarettes that we conducted in 1998 in a European city based on a quota sample of $n=421$. In a self-administered questionnaire, each respondent provided up to three the most important attributes of cigarettes. Then, he/she defined up to three perceived consequences resulting from each attribute. Finally, he/she identified up to three reasons why each of these consequences was important to him/her. The laddering part of the questionnaire, based on the format introduced, for the first time (to the best of our knowledge), by Mount and Kaciak, ${ }^{8}$ is presented in Figure 1.

Two independent judges classified these categories into seven attribute, eight benefit and seven value categories. Following the content analysis, all associations elicited from the questionnaires were structurally analysed in order to identify the linkages among the attributes $\left(a_{i}=7\right)$, the associated consequences $\left(c_{j}=8\right)$ and the underlying values $\left(v_{k}=7\right.$; hence, $\left.q=a_{i}+c_{j}+v_{k}=22\right)$. We found $P=1,828$ ladders that formed a $1,828 \times 22$ ladder matrix $L$, which was, subsequently, converted into a $22 \times 22$ SIM.

\section{ANALYSING MEC DATA}

MEC data have been analysed in the literature in a variety of ways. In this paper, we divide them into deterministic and probabilistic methods.

\section{Deterministic methods}

Most laddering studies have used deterministic methods. We classify them into two groups: methods applied to a SIM and methods applied to a ladder matrix $(L)$. 


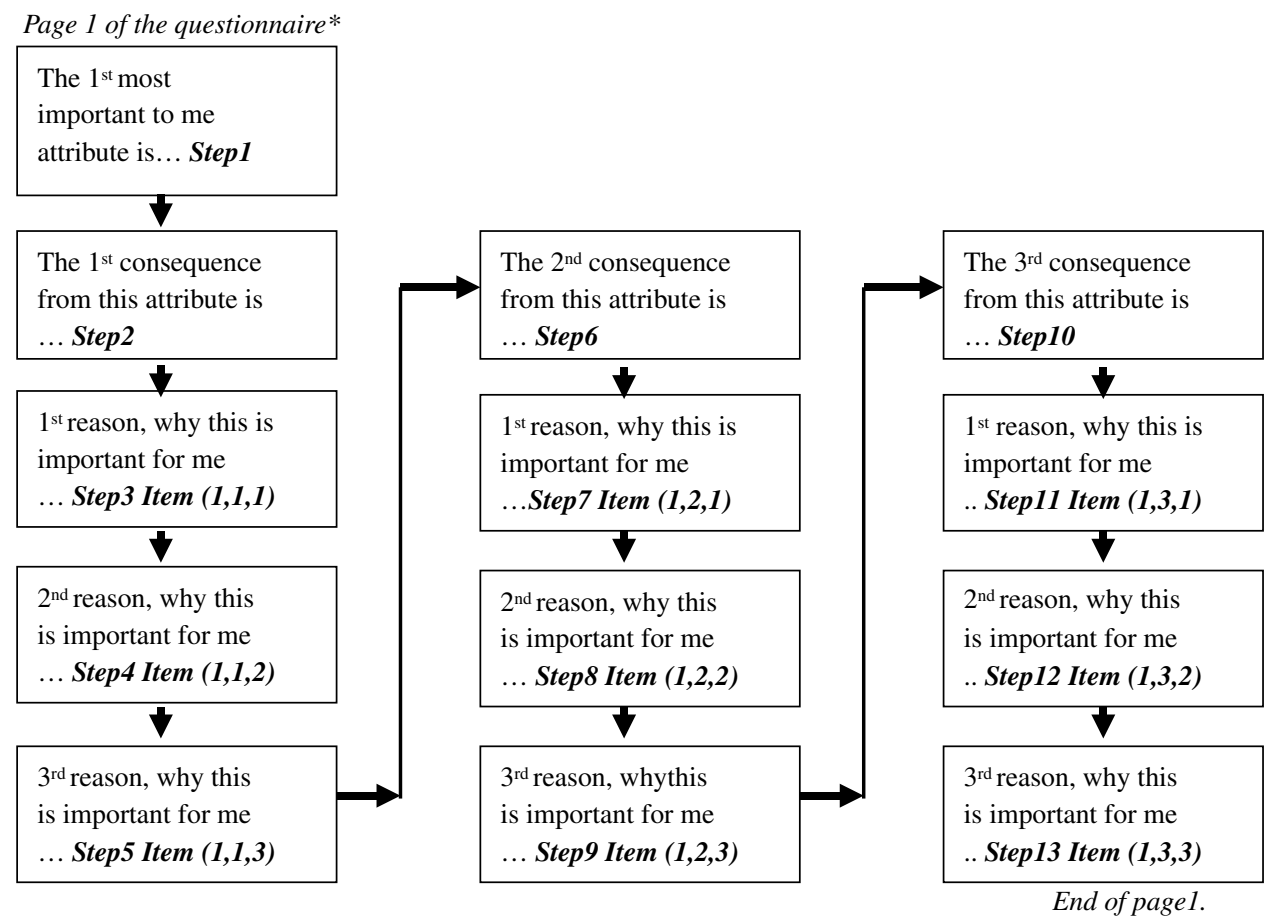

Figure 1: The laddering part of the questionnaire. *The same format will be repeated for the second and the third attributes, on pages 2 and 3 of the questionnaire, respectively

\section{Methods applied to a SIM}

- Hierarchical value map (HVM): Based on a SIM, one can draw a hierarchical value map $(H V M)$, which constitutes the most popular approach to analysis of laddering data. ${ }^{9}$ The $H V M$ is a graphical representation of the most meaningful (ie, exceeding a certain cut-off level, typically $4-5$ per cent of $n$ ) relationships among the categories in the SIM. For illustrative purposes, we present in Figure 2 the HVM obtained in our study of smokers' perceptions of cigarettes.

- Cluster analysis of a SIM: Identifying the dominant clusters of $A, C$ and $V$ categories has been proposed as an alternative to the $H V M{ }^{10}$

\section{Data-reduction methods applied directly to a ladder matrix $L$}

- Correspondence analysis (CA) and multiple correspondence analysis (MCA): CA and MCA are a natural choice for the laddering data because of their binary nature. ${ }^{11,12,13}$ Both methods produce coordinates of the laddering categories in a multidimensional space.

- Nonlinear canonical correlation analysis NCCA:Valette-Florence ${ }^{14}$ applied this method to a ladder matrix $L$ expanded by observations representing the respondent's country. The method produces coordinates of the variables' centroids in a multidimensional space.

\section{Probabilistic methods}

Probabilistic methods have been applied, to the best of our knowledge, in only two laddering studies.

\section{Multidimensional scaling of the matrix of probabilities of inter-item similarity}

Aurifeille and Valette-Florence ${ }^{15}$ have proposed an alternative to the $H V M$ method for uncovering dominant MEC from laddering data. Their procedure involves three steps: (i) calculate hypergeometrical probabilities of links between pairs of laddering items; (ii) perform nonmetric MDS on the resulting matrix of probabilities and (iii) apply a clustering procedure to the 
coordinates of the laddering categories in the MDS space.

\section{Probabilistic segmentation model} ter Hofstede et al. ${ }^{16}$ have proposed another approach alternative to the HVM - a method of constructing probabilistic means-end map segments. They have calculated the probabilities of a respondent choosing a link between an attribute and a consequence or between a consequence and a value, and then have used the strengths of these links as the basis for segmentation.

The diversity of the above listed methods suggests that there is no clear agreement among researchers as to which approach is more appropriate. The probabilistic methods, although very promising, have not been used by many researchers, probably due to lack of easily accessible software. In this paper, we will therefore focus on the deterministic methods only.

\section{RESEARCH ISSUES AND METHODOLOGY}

\section{Hierarchical value map (HVM)}

The most popular method of analysis of laddering data, beyond question, is the hierarchical value map. ${ }^{9}$ The $H V M$ graphically displays the data contained in the SIM in their least distorted form (the degree of distortion will only depend on the choice of the cut-off level). Other methods introduce certain transformations to the data, for example, dimensionality reduction. On the other hand, these methods, unlike the HVM, can accommodate the respondent/product characteristics necessary for segmentation purposes. In this paper, we will determine which method produces the least distorted picture of the structure in the HVM. The quality of a laddering structure emerging from the analysis will be evaluated from two perspectives: (i) its ability to preserve associations within all possible pairs of laddering categories observed in the input data and (ii) its ability to preserve associations within all possible triads of laddering categories. These two measures are comparable to the structure measure of the MEC network used by ter Hofstede et al. ${ }^{17}$

\section{Data-reduction methods}

We will consider the following three datareduction methods (to the best of our knowledge, $M D S \_S I M$ and $C C A \_L$ have never been reported in the literature).

\section{Multidimensional scaling of SIM (MDS_SIM)}

The only method, other than the HVM, that has been applied to a SIM is cluster analysis. ${ }^{10}$ Because the elements of SIM can be treated as proximities (similarities) between different laddering categories (the larger the element in a SIM, the closer the two corresponding categories), the use of the MDS is also a natural choice for this type of data.

\section{Multiple correspondence analysis of $L$ (MCA_L)}

Valette_Florence ${ }^{14}$ has applied nonlinear canonical correlation analysis (NCCA) to a ladder matrix $L$, expanded by observations representing the respondent's country. Such expanded matrix $L$ is a natural choice for the NCCA if at least one of the sets contains more than one variable. When each of the sets has only one variable, the NCCA and MCA produce identical results. Since the ladders in our matrix $L$ have only three levels, the two methods are equivalent.

\section{Canonical correlation analyses of $L$ (CCA_L)}

When only two sets are involved, and at least one of them has more than one variable, NCCA becomes canonical correlation analysis. We assigned the three variables $(A, C$ and $V)$ in $L$, into two sets so that one set included one variable, and the other - two variables, and analysed each pair of the two sets with the CCA.

\section{Comparing the HVM and the data-reduction methods}

In order to compare the HVM and the datareduction methods, we propose two similarity measures: the strength of connections within pairs $\left(A_{i}, C_{j}\right),\left(A_{i}, V_{k}\right)$ and $\left(C_{j}, V_{k}\right)$, and the strength of connections within triads $\left(A_{i}, C_{j}, V_{k}\right)$. 


\section{The strength of connections within pairs $\left(A_{i}, C_{j}\right),\left(A_{i}, V_{k}\right)$ and $\left(C_{j}, V_{k}\right)$}

For each of the three methods, we measured the similarity of any two laddering categories as the Euclidean distance between the corresponding two vectors of coordinates produced by this method in a six-dimensional space (we chose six dimensions based on the scree plot in factor analysis of the correlation matrix of the 22 laddering categories in $L$ ).

The smaller the distance between two categories, the more similar they are across all the ladders. These distances were subsequently compared to the occurrences of all possible bilateral linkages among $A$ 's, $C$ 's and $V$ s. Such occurrences can be found in the SIM, where each element represents the number of times a category (attribute, consequence or value) leads to another category. Ideally, large elements in the SIM should match small distances in the space.

We evaluated the degree of match between the distances and the (converted to dissimilarities) elements of SIM by calculating the corresponding Pearson correlation coefficients (Table 1). The correlations indicate that the bilateral associations between the categories are best represented by the MDS of the SIM. The results of both the MCA and the CCA of $L$ match these bilateral associations with a slightly lesser precision.

\section{The strength of connections within triads $\left(A_{i}, C_{j}, V_{k}\right)$ of laddering items}

For each of the three methods, we measured the similarity of any three categories as the (Euclidean) perimeter of a triangle created by the corresponding three vectors of coordinates produced by the method in a six-dimensional space. The smaller the perimeter of a triangle formed by three categories, the more similar they are across all ladders. We then compared these perimeters to the occurrences of all possible trilateral linkages among $A$ 's, $C$ 's and $V$ s. Such trilateral linkages are not present in the SIM, for it shows only bilateral connections between the categories. In this paper, we propose a method for determining a matrix that displays occurrences of all the trilateral linkages. We term this matrix a summary ladder matrix or SLM. To the best of our knowledge, this matrix has never been reported in the literature.

\section{Constructing a summary ladder matrix (SLM)}

A $S L M$ can be obtained as follows:

Step 1: Sort the rows of $L$ by any of the category concepts, that is, either by attributes, consequences or values. Let us assume that the consequence categories have been chosen as a sorting criterion. (A choice of attributes or values will generate the same outcome.) As a result, one obtains a sorted $L$ with the first group of rows representing all the ladders that include the first consequence category, followed by all the ladders with the second consequence category, etc. Let us assume that there are $p_{j}$ ladders containing the consequence category $C_{j}(j=1$, $\left.2, \ldots, c_{j}\right)$, so that $p_{1}+p_{2}+\cdots+p_{c j}=p$. The sorted $L$ can therefore be represented as follows (for the sake of space, we represent $L$ in the transposed form): $L^{\prime}=\left[\begin{array}{lllll}L_{1}{ }^{\prime} & L_{2}{ }^{\prime} & \ldots & L_{c j}\end{array}\right]$, where $L_{j}=\left[A_{j} C_{j} V_{j}\right]$, and where $A_{j}, C_{j}$ and $V_{j}$ are sub-matrices of $L_{j}$ with dimensions $\left(p_{j} \times a_{i}\right),\left(p_{j} \times c_{j}\right)$ and $\left(p_{j} \times v_{k}\right)$, respectively.

Table 1: Pearson correlation coefficients between the distances and the elements of SIM

\begin{tabular}{llllllll}
\hline & SIMdissim & MDS_SIM & MCA_L & CCA_A/CV & CCA_C/AV & CCA_V/AC & MDS_L \\
\hline SIMdissim & 1 & 0.895 & 0.818 & 0.760 & 0.797 & 0.736 & 0.534 \\
MDS_SIM & 0.895 & 1 & 0.833 & 0.758 & 0.793 & 0.719 & 0.653 \\
MCA_L & 0.818 & 0.833 & 1 & 0.786 & 0.952 & 0.890 & 0.574 \\
CCA_A/CV & 0.760 & 0.758 & 0.786 & 1 & 0.736 & 0.633 & 0.496 \\
CCA_CIAV & 0.797 & 0.793 & 0.952 & 0.736 & 1 & 0.859 & 0.520 \\
CCA_VIAC & 0.736 & 0.719 & 0.890 & 0.633 & 0.859 & 1 & 0.456 \\
MDS_L & 0.534 & 0.653 & 0.574 & 0.496 & 0.520 & 0.456 & 1 \\
\hline
\end{tabular}

Note: All the correlations are significant at the 0.01 level (two-tailed). 
Table 2: Pearson correlation coefficients between the triangles' perimeters and the elements of SLM

\begin{tabular}{llllllll}
\hline & SLMdissim & MDS_SIM & MCA_L & CCA_A/CV & CCA_C/AV & CCA_V/AC & MDS_L \\
\hline SLMdissim & 1 & 0.714 & 0.591 & 0.558 & 0.573 & 0.525 & 0.472 \\
MDS_SIM & 0.714 & 1 & 0.816 & 0.753 & 0.778 & 0.716 & 0.681 \\
MCA_L & 0.591 & 0.816 & 1 & 0.766 & 0.955 & 0.917 & 0.579 \\
CCA_A/CV & 0.558 & 0.753 & 0.766 & 1 & 0.725 & 0.707 & 0.538 \\
CCA_C/AV & 0.573 & 0.778 & 0.955 & 0.725 & 1 & 0.865 & 0.530 \\
CCA_VIAC & 0.525 & 0.716 & 0.917 & 0.707 & 0.865 & 1 & 0.476 \\
MDS_L & 0.472 & 0.681 & 0.579 & 0.538 & 0.530 & 0.476 & 1 \\
\hline
\end{tabular}

Note: All the correlations are significant at the 0.01 level (two-tailed).

Step 2: Multiply $V_{j}^{\prime}$ by $A_{j}, j=1,2, \ldots, c_{j}$. Each $v_{k}$ $\times a_{i}$ matrix $V_{j}^{\prime} A_{j}$ represents a number of ladders between all attribute and value categories that pass through the consequence category $C_{j}$.

Step 3: Stacking the matrices $V_{j}^{\prime} A_{j}(j=1,2, \ldots$, $\left.c_{j}\right)$ on top of each other creates a $S L M$ :

$$
\begin{aligned}
& S L M= \begin{array}{l}
V_{1}{ }^{\prime} A_{1} \\
V \ldots \ldots
\end{array} \\
& V c_{j}{ }^{\prime} A c_{j}
\end{aligned}
$$

Since each matrix $V_{j}^{\prime} A_{j}$ is a $v_{k} \times a_{i}$ matrix, and there are $c_{j}$ such matrices, the dimensions of a $S L M$ are $\left(c_{j} \times v_{k}\right) \times a_{i}$. Elements of a summary ladder matrix represent numbers of times each three categories $\left(A_{i}, C_{j}, V_{k}\right), i=1,2, \ldots, a_{i} ; j=1,2, \ldots, c_{j}$; $k=1,2, \ldots, v_{k}$, have been linked together by respondents across all laddering interviews. Matrices SLM and SIM are related. One can obtain the SIM from the SLM but the reverse procedure is impossible.

We evaluated the degree of match between the perimeters of the triangles and the (converted to dissimilarities) elements of the SLM by calculating the corresponding correlations, presented in Table 2. The correlations indicate that the trilateral connections between the categories are, again, best represented by the MDS of the SIM.

\section{SUMMARY OF THE RESULTS}

Based on the above analyses of bilateral and trilateral associations between the laddering categories, we conclude that the MDS of the SIM produces results that best match the structure of the underlying $H V M$.

This result is important because supplementing the $H V M$ with MDS of the SIM permits market segmentation. For this purpose, we expanded the ladder matrix $L$ by consumer/product characteristics $(S)$ to $L_{E}=[A C V S]$, then converted it to the $S I M_{E}=L_{E}^{\prime} L_{E}$ and applied the MDS procedure. We then repeated the analyses of the bilateral and trilateral associations between the laddering categories extracted from this new MDS solution. We obtained the correlation coefficients similar to the ones found previously for the (unexpanded) SIM. This result indicates that the MDS of the $\operatorname{SIM}_{E}$ preserves the relationships among the laddering categories found by the MDS of the SIM and thus matches well the structure of the underlying HVM. The variables' MDS coordinates can then be analysed with a clustering method in order to identify market segments - the dominant associations between the MEC and the consumer/product characteristics. For example, the $H V M$ presented in Figure 2, in combination with MDS followed by a clustering method, provides an easily accessible summary of the complex network of attributes, consequences and values related to the consumers' perceptions of cigarettes. Identifying and understanding the relationships among the nodes in this $H V M$ may help to better understand the behavioural underpinnings of tobacco consumption. Such a summary, enhanced by knowledge of the consumer/product characteristics, could, for example, be valuable for those responsible for public health issues. An examination of the chains linking attributes of cigarettes, through the consequences, to the values that underlie the choice of cigarette could provide an opportunity for more precisely targeting public health initiatives. The objective would be to create persuasive communications 


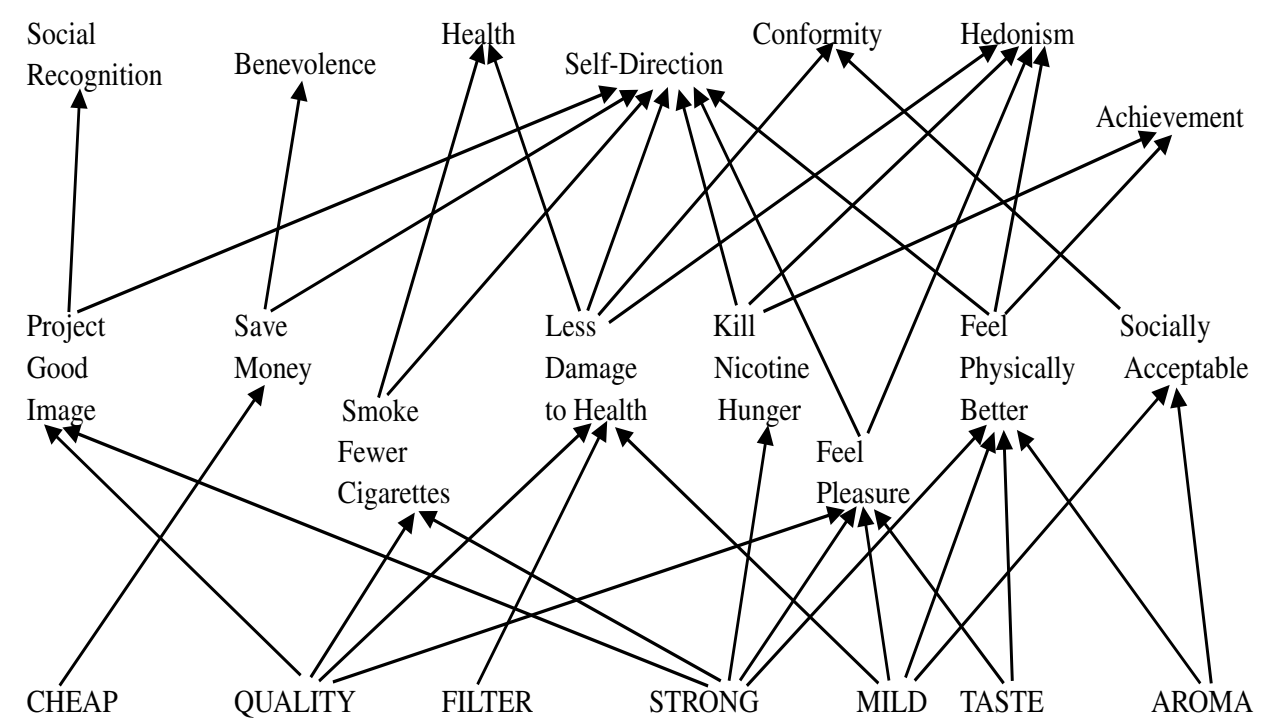

Figure 2: Hierarchical value map of smokers' perceptions of cigarettes $(n=421)$

that might discourage smoking by tapping, for example, directly into the consumers' values rather than focusing trivially on the product's attributes.

\section{DISCUSSION, CONCLUSIONS AND LIMITATIONS}

In this paper, we have evaluated the results of three data-reduction methods of analysis of laddering data, comparing them to the HVM, the most popular technique in the laddering research. To the best of our knowledge, two of these methods (the MDS of the SIM and the CCA of $L)$, have not been used for analysis of laddering data before. The HVM, although very popular, has one significant limitation. It does not enable the inclusion of the respondent/product measures and, therefore, does not allow for market segmentation. On the other hand, it represents the data in their least distorted form, which depends only on the choice of the cut-off level. The data-reduction techniques have exactly the opposite features. They can accommodate the respondent/product characteristics, necessary for segmentation purposes; however, they introduce certain transformations to the data. Therefore, it makes sense to seek a data-reduction technique that would minimise the distortions of the original data, that is, would match the laddering structure in the HVM in the best possible way. This was the objective of this paper.

In order to evaluate these methods, we introduced two similarity measures, comparable to the structure measure of the MEC network used by ter Hofstede et al. ${ }^{17}$ The first similarity measure involved pairs of the laddering categories, and was based on the summary implication matrix (SIM). The second measure focused on triads of the laddering categories, and was based on the summary ladder matrix (SLM), that we have presented, for the first time, in this paper.

We have found that although the best results can be obtained with the MDS of the SIM, other data-reduction techniques such as MCA or CCA, applied directly to the ladder matrix $L$, produce comparable results. Our finding has practical implications for those who might hesitate as to which method they should use for analysis of what form of laddering data. Ideally, one could try all the approaches mentioned above and interpret only patterns present across all the solutions.

Our findings suggest that for market segmentation purposes, the $H V M$ can be used in tandem with $M D S$ of the summary implication matrix $\left(S I M_{E}\right)$ expanded by consumer/product characteristics. Other data-reduction techniques (eg, MCA, NCCA or CCA), applied directly to 
the expanded ladder matrix $L_{E}=[A C V S]$, although (based on our results) somewhat inferior to the MDS of $S I M_{E}$, may also shed some additional light on the relationships among the laddering categories and the consumer/product characteristics. We also suggest that one should always perform data-reduction procedures, such as MCA, NCCA and/or CCA, on the consumer/ product characteristics $(S)$ alone. The patterns emerging from such analyses should be matched against the patterns found in the previous (aggregated) analyses. Only the patterns that are present in all outcomes may be considered for segmentation purposes.

The summary ladder matrix (SLM) that we have introduced in this paper contains more information than the traditional summary implication matrix (SIM). It provides information not only about which pairs of the laddering categories were elicited from the respondents but also which triads of categories were formed. We point to an interesting additional benefit stemming from the knowledge of the SLM. Based on this matrix, we can calculate a ratio of the number of $A-C-V$ ladders represented in the $H V M$ to the total number of ladders. This ratio, which we introduce for the first time in this paper, and name the $S L M$ ratio, may be used together with the traditional SIM ratio ${ }^{9}$ to evaluate the quality of the hierarchical value map. In many laddering studies, the reported SIM ratio was rather low (eg, 44-54 per cent, ${ }^{18} 44-51$ per cent, ${ }^{19} 47$ per cent $^{20}$ ), which might create a rather pessimistic impression of the quality of the resulting $H V M$ s. This pessimistic impression may be somewhat softened if the quality of the HVM is measured with the SLM ratio. For example, in the study described in this paper, the traditional SIM ratio was 50.8 per cent, whereas the SLM ratio was found to be 79.3 per cent! The SLM ratio is a measure of the quality of the $H V M$ that focuses on the original ladders in the SLM rather than on the associations in the SIM, and as such provides additional information about the accuracy of the map.

Our study has some limitations. First, we have based our conclusions on the results from the analyses of only one data set (in future research, these methods should be applied to a number of synthetic ladder matrices $L$ generated in a Monte Carlo study). Secondly, we have focused only on deterministic methods for analysing laddering data (future research is required that will also involve probabilistic methods in the comparative analysis).

\section{References}

1 Gutman, J. (1982) 'A means-end chain model based on consumer categorization process', Journal of Marketing, Vol. 46, No. 2, pp. 60-72.

2 Gutman, J. (1984) 'Analyzing consumer orientations toward beverages through means-end chain analysis', Psychology and Marketing, Vol. 1, No. 3/4, pp. 23-43.

3 Howard, J. A. (1977). Consumer Behavior: Application and Theory, McGraw-Hill, New York.

4 Newel, A. and Simon, H. A. (1972). Human Problem Solving, Prentice Hall, Englewood Cliffs, NJ.

5 Olson, J. C. and Thomas, J. R. (1983) 'Understanding Consumers' Cognitive Structures: Implications for Marketing Strategy', in Percy, L and Woodside, A.G. (eds), Advertising and Consumer Psychology, Lexington Books, Lexington, MA, pp. 77-90.

6 Young, S. and Barbara, F. (1975) 'Using the benefit chain for improved strategy formulation', Journal of Marketing, Vol. 39, pp. $72-74$.

7 Olson, J. C. and Thomas, J. R. (2001) 'The means-end approach to understanding consumer decision making', in Reynolds, Thomas J. and Olson, Jerry C (eds), Understanding Consumer Decision Making: The Means-End Approach to Marketing and Advertising Strategy, Lawrence Earlbaum Associates, Mahwah, New Jersey, pp. 3-20.

8 Mount, J. and Eugene, K. (1993) 'Transforming the laddering technique into a research tool for everyday use', Proceedings of the ASAC 1993 Conference, pp. 205-217.

9 Reynolds, T. J. and Gutman, J. (1988) 'Laddering theory, method, analysis, and interpretation', Journal of Advertising Research Vol. 28, No. 1, pp. 11-31.

10 Klenosky, D. B., Gengler, C. E. and Mulvey, M. S. (1993) 'Understanding the factors influencing ski destination choice: A means-end analytic approach', Journal of Leisure Research, Vol. 25, pp. 362-379.

11 Valette-Florence, P. and Rapacchi, B. (1991) 'Improvements in means-end chain analysis: Using graph theory and correspondence analysis', Journal of Advertising Research, Vol. 31, No. 1, pp. 30-45.

12 Nielsen, N. A., Bech-Larsen, T. and Grunert, K. G. (1998) 'Consumer purchase motives and product perceptions: A laddering study on vegetable oil in three countries', Food Quality and Preference, Vol. 9, No. 6, pp. 455-466.

13 Grunert, K. G., Lahteenmaki, L., Nielsen, N. A., Poulsen, J. B., Ueland, O. and Astrom, A. (2001) 'Consumer perceptions of food products involving genetic modification - Results from a qualitative study in four Nordic countries', Food Quality and Preference, Vol. 12, pp. 527-542. 
14 Valette-Florence, P. (1998) 'A causal analysis of means-end hierarchies in a cross-cultural context: Methodological refinements', Journal of Business Research, Vol. 42, pp. 161-166.

15 Aurifeille, J. M. and Valette-Florence, P. (1995) 'Determination of the dominant means-end chains: A constrained clustering approach', International Journal of Research in Marketing, Vol. 12, pp. 267-278.

16 ter Hofstede, F., Steenkamp, J. B. E. M. and Wedel, M. (1999) 'International market segmentation based on consumer-product relations', Journal of Marketing Research, Vol. 36, pp. 1-17.

17 ter Hofstede, F., Audenaert, A., Steenkamp, J. B. E. M and Wedel, M. (1998) 'An investigation into the association pattern technique as a quantitative approach to measuring means-end chains', International Journal of Research in Marketing, Vol. 15, pp. 37-50.
18 Sorensen, E., Grunert, K. G. and Nielsen, N. A. (1996) 'The impact of product Experience, Product Involvement and Verbal Processing Style on Consumers' Cognitive Structures with Regard to Fresh Fish', Working Paper No. 42, October, The Aarhus School of Business, Aarhus, Denmark.

19 Botschen, G. and Hemetsberger, A. (1998) 'Diagnosing meansend structures to determine the degree of potential marketing program standardization', Journal of Business Research, Vol. 42, pp. 151-159.

20 Miele, M. and Parisi,V. (2000) 'Consumer concerns about animal welfare and the impact on food choice', Italian Report on Laddering Interviews', FAIR project CT98-3678, Department of Agricultural Economics, University of Pisa. 